\title{
Chapter 3 \\ Execution and Cancellation Lifetimes in Foreign Currency Market
}

\author{
Jean-François Boilard, Hideki Takayasu, and Misako Takayasu
}

\begin{abstract}
We analyze mechanisms of foreign currency market order's annihilation with a focus on the lifetime of these orders. Limit orders submitted in this market are approximately executed according to the random walk theory. In consequence, the distribution of execution lifetime can be approximated by a power law with exponent $1 / 2$. Alternatively, limit orders submitted in foreign currency markets are roughly cancelled according to a mixed distribution; as a random walk with a tail following a power law. The cancellation lifetime distribution can be approximated by using a scaling relationship between the distance from mid-price and the random walk theory. In addition, we introduce the concept that market participants cancel orders depending on the market price's movement which is represented as the movement of the mid-price. Taking into consideration market conditions when orders have been injected, market participants do not have symmetric decision rules. This behavior could at least partially explain the shape of price change distribution.
\end{abstract}

\footnotetext{
J.-F. Boilard $(\square) \bullet$ M. Takayasu

Department of Computational Intelligence and Systems Science, Interdisciplinary Graduate School of Science and Engineering, Tokyo Institute of Technology, 4259-G3-52, Nagatsuta-cho, Midori-ku, Yokohama 226-8503, Japan

e-mail: boilard.j.aa@m.titech.ac.jp; takayasu@dis.titech.ac.jp

H. Takayasu

Sony Computer Science Laboratories, 3-14-13, Higashigotanda, Shinagawa-ku, Tokyo 141-0022, Japan

Meiji Institute of Advanced Study of Mathematical Sciences, Meiji University, 4-21-1 Nakano, Nakano-ku, Tokyo 164-8525, Japan

Department of Computational Intelligence and Systems Science, Interdisciplinary Graduate School of Science and Engineering, Tokyo Institute of Technology, 4259-G3-52, Nagatsuta-cho, Midori-ku, Yokohama 226-8503, Japan

e-mail: takayasu@csl.sony.co.jp
}

(C) The Author(s) 2015

H. Takayasu et al. (eds.), Proceedings of the International Conference on Social

Modeling and Simulation, plus Econophysics Colloquium 2014, Springer

Proceedings in Complexity, DOI 10.1007/978-3-319-20591-5_3 


\subsection{Introduction}

Financial market movements have been studied firstly by Bachelier who described price movements as a random walk [1]. Since then, many researchers studied the microstructure of markets [2-4] and more recently some of them have focused on order book fluctuations using physics methodology [5-7]. Availability of new detailed database help researchers precisely describe the impacts of some characteristics on the order book even if orders do not directly contribute to execution.

In addition, some researchers describe order injection deal and cancellation from a physics viewpoint. In a paper appeared in 2014, Yura et al. studied the correlation between layers in the order book and the market price movement based on the analogy with the colloidal motion in water molecules [8].

Recently, a high proportion of market transactions are done by automated traders (financial algorithms) which increase the reaction speed in the market as a whole [9]. Regulators focus on analysing lifetime of orders - the amount of time spent in the market before annihilation - to understand the impact of market speed on the stability of the financial market $[10,11]$. An extraordinary event in May 2010, better known as the "flash crash", puts light on direct impact of cancellation orders on market volatility [12]. Actually, governmental organizations recognize that cancellation orders should be studied more carefully concerning cancellation lifetime [13].

The next section details the database used for this study. In Sect.3.3 we describe lifetime statistical properties of annihilated limit orders with a focus on the relationship between executed and cancelled orders. Section 3.4 describes how market participants cancel their orders considering movement in market price. The final section contains a summary.

\subsection{Description of the Database}

We use a special database of Electronic Broking System (EBS) which contains identifications of every order. The database is from March 13th 21:00 (GMT) to March 18th 21:00 (GMT) 2011, and contains information about injected and annihilated orders with minimal tick time of $1 \mathrm{~ms}$. This foreign exchange market is open $24 \mathrm{~h}$ per day during weekdays.

Traders must have a direct access to the market server via a private network and most of them are either banks or financial institutions. The majority of participants use financial algorithms for trading activity. In consequence, EBS market implements a minimum cancellation time rule of $250 \mathrm{~ms}$. Then, it is not possible for participants to cancel a previous submitted order if the quote life is lower than $250 \mathrm{~ms}$. As soon as the life quote is higher than this minimum, it is free for participants to do as they want. 
Table 3.1 Details concerning limit orders

\begin{tabular}{l|c|l|l}
\hline Currency & Submitted & Cancelled & Executed \\
\hline USD/JPY & $2,695,128$ & $2,436,385(90.4 \%)$ & $258,743(9.6 \%)$ \\
\hline EUR/JPY & $1,481,285$ & $1,439,801(97.2 \%)$ & $41,484(2.8 \%)$ \\
\hline EUR/CAD & 370,903 & $370,717(99.9 \%)$ & $186(0.1 \%)$ \\
\hline
\end{tabular}

The order book is initialized at the beginning of the week and similarly all orders remaining at the end of the week are deleted. This market is described as overthe-counter (OTC). It means that market participants must already have a credit agreement with other participants before they transact with each other. If this kind of agreement does not already exist, it is not possible for them to make a transaction. In other words, it is possible to see a buying order at a higher price than a selling order (negative spread) if there is no credit agreement between these market participants.

The EBS database contains forty-eight currencies but our study focuses on two of them: USD/JPY and EUR/JPY. Both are very liquid currencies in EBS market. During our studied period, the minimal tick (pip) for USD/JPY and EUR/JPY is 0.001 yen.

Table 3.1 describes the occurrence of limit orders submitted during our studied period and the high proportion of cancelled orders is explained by the type of traders within this market. In fact, over $90 \%$ of traders are automated softwares (algorithmic trading) and most of them are market makers. We can define market making strategy as a provider of limit orders in the electronic order book which in exchange is remunerated with the spread charged between bid and ask orders and possibly a rebate fee (if available). In consequence, they have a high incentive to protect themselves against execution risk (risk to see their limit orders executed at the worst time). As cancelling and submitting orders do not incur costs, they often update their orders depending on market conditions (volatility, news, etc.) which increase significantly the proportion of cancelled orders [14].

The most traded currency in EBS database is the USD/JPY in term of submitted limit orders and globally $90 \%$ of all limit orders are ultimately cancelled. Another widely traded currency is EUR/JPY especially for triangular arbitrage. The percentage of cancellations increase when the currency is less liquid, for example, almost all submitted orders in EUR/CAD currency pair are cancelled which makes the proportion of cancelled orders extremely high $(99.9 \%)$.

In this current research, we use the notation $b(t)$ as the best bid price at time $t$ and $a(t)$ as the best ask (offer) price at time t. The mid-price $m(t)$ is the average between the best bid and best ask price: $m(t)=[b(t)+a(t)] / 2$. Figure 3.1 represents histogram of initial distance of orders in pips from the mid-price. The black histogram is the frequency of submitted orders at this initial distance and the grey histogram is the frequency of submitted orders at this initial distance knowing they will be executed.

We can observe that orders initially submitted around mid-price have higher chances to be executed than orders far from these prices. In addition, the proportion of executed orders decreases for orders far from the mid-price. In our research, we 
Fig. 3.1 Semi-log frequency plot of initial distance of limit orders from the mid-price of USD/JPY currency pair. Grey histogram represents all limit orders and black histogram represents limit orders to be executed

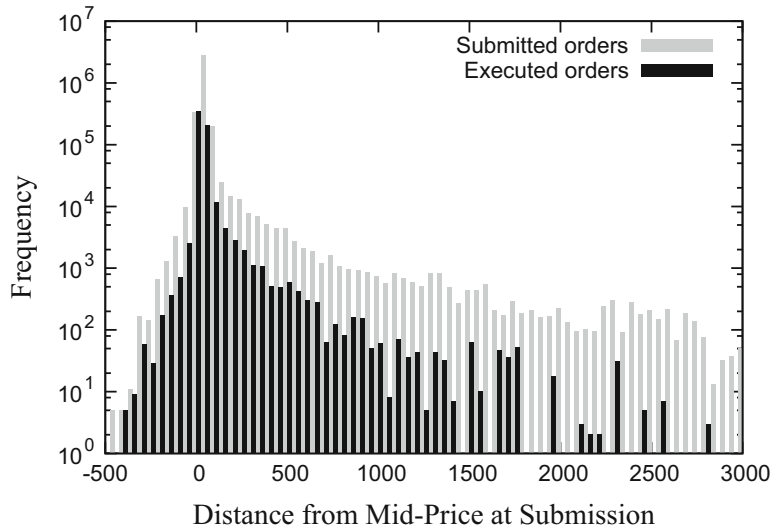

analyze both bid and ask orders symmetrically as they have similar properties in our market.

\subsection{Execution and Cancellation Lifetimes}

The life of an order starts when it is injected in the electronic order-book. Similarly, this order's life ends when it is finally executed or cancelled (annihilation process). The time between the beginning and ending of this order is defined as lifetime. In this section we focus on statistical properties of execution and cancellation lifetimes.

\subsubsection{Execution Lifetime}

An electronic order-book is filled by limit orders. A transaction occurs when a buying order (bid) is at a price equal or higher than a selling order price (ask or offer). In addition, both market participants must already have a credit agreement to fulfil the transaction. Figure 3.2 represents the cumulative distribution of lifetime of limit orders that are executed.

Execution lifetime $\left(t_{L}\right)$ can be described as the time between the injection of the order and execution. Assuming the market price is approximated by a random walk and knowing a typical injection is close to the market price, the lifetime is estimated by a recurrence time of random walk [8]:

$$
P\left(>t_{L}\right) \propto t_{L}^{-\frac{1}{2}}
$$


Fig. 3.2 Log-log cumulative distribution of execution and black dotted lines respectively represent USD/JPY and EUR/JPY currency pairs orders lifetime. The grey bold

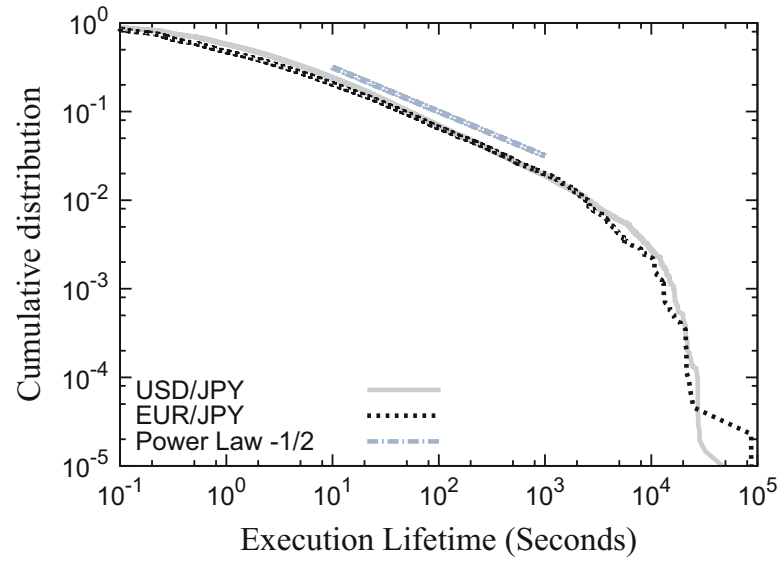

Fig. 3.3 Log-log cumulative distribution of the initial distance in pips from mid-price when orders are executed. The grey bold and black dotted lines respectively represent USD/JPY and EUR/JPY currency pairs

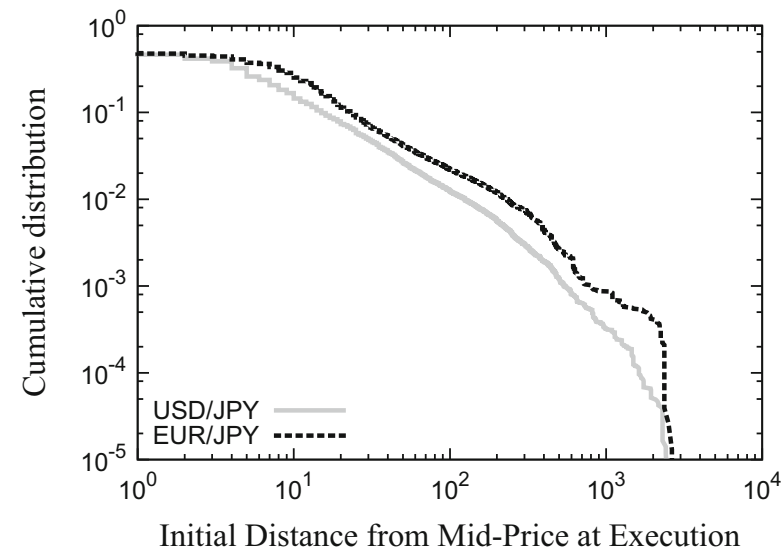

As confirmed in Fig. 3.2 lifetimes of execution orders in EBS foreign market are approximately following the random walk theory both for USD/JPY and EUR/JPY currency pairs.

Figure 3.3 represents the distance from mid-price at injection of orders later annihilated via execution. There are a lot of orders initially injected close from market price and ultimately executed.

\subsubsection{Cancellation Distance and Lifetime}

In this foreign currency market, participants can always decide to cancel their initial limit orders if their lifetime is longer or equal to $250 \mathrm{~ms}$. Contrary to execution orders, cancellation orders are annihilated at a certain distance from the mid-price. 
Fig. 3.4 Log-log cumulative distribution of cancellation orders lifetime. The grey bold and black dotted lines respectively represent USD/JPY and EUR/JPY currency pairs

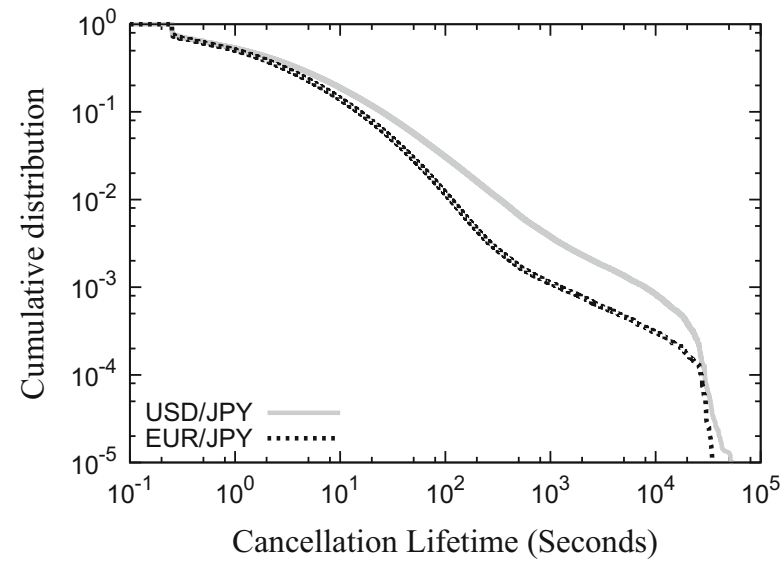

Fig. 3.5 Log-log cumulative distribution of distance in pips from mid-price when orders are cancelled. The grey bold and black dotted lines respectively represent USD/JPY and EUR/JPY currency pairs

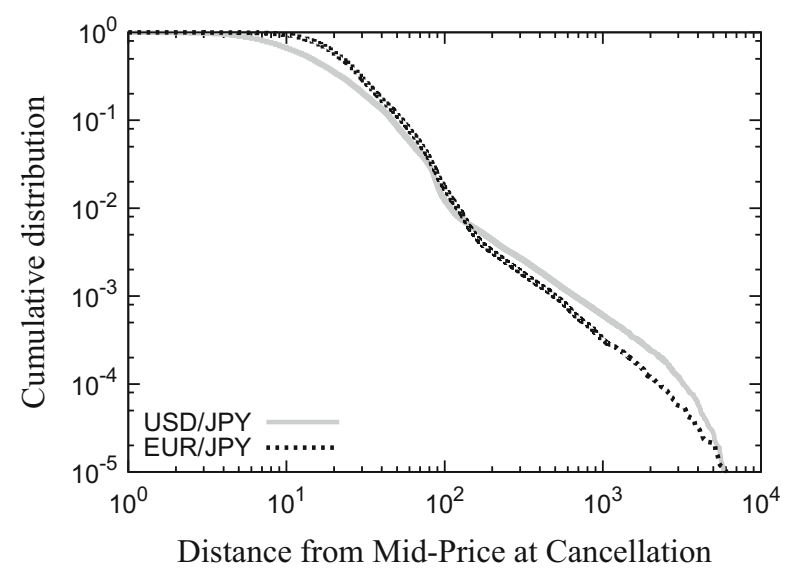

Many reasons can push a market participant to cancel an order such as being more or less aggressive with the price of his limit order.

Figure 3.4 represents the cancellation lifetime $\left(T_{L}\right)$ cumulative distribution. In both currency pairs, more than $20 \%$ of orders are cancelled at the minimum allowed cancellation lifetime $(250 \mathrm{~ms})$. Furthermore, the cancellation lifetime distribution bends in comparison to execution lifetime. In other words, there is significantly more orders staying a long time in the electronic order book and ultimately cancelled.

In Fig. 3.5 we analyze the cumulative distribution of cancellation orders from the mid-price $[m(t)]$. For small distance from mid-price represented from $10^{0}$ to $10^{1}$, both currencies initially have a plateau because of the spread between bid and ask orders. USD/JPY and EUR/JPY generally have a spread of approximately ten pips. If the order is closer from the mid-price, the chance to be executed is greater. Inversely, there are lower chances to be executed if orders are far from the midprice. The distribution of the distance from mid-price at cancellation (Fig. 3.5) is 
significantly different from the distribution of the initial distance from mid-price at execution (Fig. 3.3).

\subsubsection{Power Law Relation Between Execution and Cancellation Lifetimes}

In Sects. 3.3.1 and 3.3.2 we described distributions of execution and cancellation lifetimes. In this section, we show there is a relationship between both distributions.

In Fig. 3.6 cumulative distribution of lifetime of annihilated events are plotted for cancellation and execution in log-log scale. Difference between execution and cancellation lifetimes observed in this figure is mostly due to the non-constant proportion between execution and cancellation of orders. In consequence, orders far from the mid-price takes longer time before being annihilated via cancellation. Limit orders far from the mid-price are often referred as extreme orders. EBS market is open from Sunday 21:00 (GMT) to Friday 21:00 (GMT) which means the maximum cancellation and execution lifetime is $\sim 6 \times 10^{5} \mathrm{~s}$. In other words, there is a natural cutoff in the lifetime of orders in our database. At the end of the week (Friday 21:00 GMT), all orders remaining in the order book are deleted and those deleted orders are not include in cancellation statistics. New injections of orders to fill the order book are only allowed on the next Sunday 21:00 GMT.

In the electronic order book, market participants initially submit their orders at a certain distance $(\bar{r})$ from the market price. If the order is closer from market price, market participants will assume there is a higher probably to see their orders executed in a shorter period of time $(T)$. Inversely, orders far from the market price will take longer time before being executed. When the market participants inject their orders, they are aware of this fact and will cancel their orders if the initial scenario does not hold anymore. If this is the case, they might choose to cancel and inject their orders again at a different price. Considering this mechanism, it is

Fig. 3.6 Log-log cumulative distribution of annihilated orders lifetime of USD/JPY currency pair

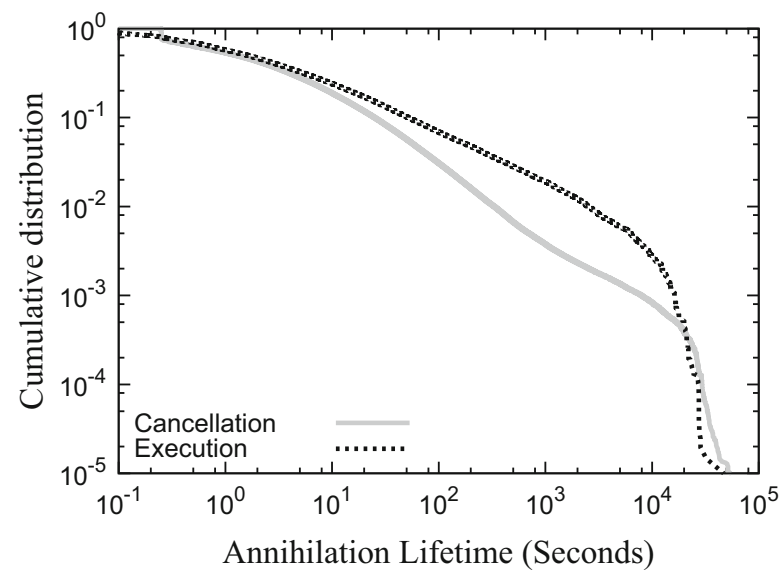


Fig. 3.7 Log-log cumulative distribution of execution orders lifetime. The grey line represents theoretical distribution estimated from Fig. 3.3 using Eq. (3.3) and the black line shows real data execution lifetime (Fig. 3.2)

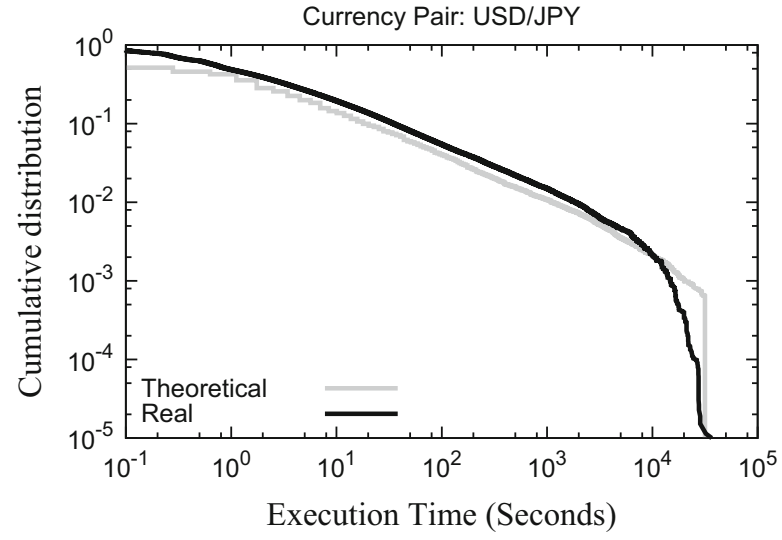

reasonable to assume the random walk theory just like the case of derivation of Eq. (3.1). We can introduce the following scaling relation:

$$
T \propto \bar{r}^{2}
$$

As demonstration of the scaling relation presenting in Eq. (3.2), we apply this relationship on the USD/JPY currency pair. Execution lifetime is approximated in using the probability distribution of the initial distance from mid-price of orders executed presented in Fig. 3.3 with the above scaling relation multiplied by a factor. Equation (3.3) is used to calculate the theoretical line from Fig. 3.3. Exempt the slower decay at the plateau on Fig. 3.7, the theoretical and real data lines fit well.

$$
T=0.07 \times \bar{r}^{2}
$$

To continue, we use the same methodology to approximate the cancellation lifetime of USD/JPY currency pair. Cancellation lifetime is approximated in using the probability distribution of the distance from mid-price of orders cancelled presented in Fig. 3.5 with the scaling relation (Eq. (3.2)) multiplied by a factor. Equation (3.4) is used to calculate the theoretical line. Exempt the slower decay at the plateau on Fig. 3.8, the theoretical and real data lines agree approximately.

$$
T=0.01 \times \bar{r}^{2}
$$

If the distance from the mid-price at cancellation follows a power-law such as the interval $10^{2}$ to $10^{4}$ in Fig. 3.8, we can directly link each other exponent. We define two power law exponents, $\alpha$ and $\delta$ respectively representing the cancellation lifetime and distance from mid-price at cancellation. For cancellation lifetime $\left(T_{L}\right)$ cumulative distribution, we have the following relationship:

$$
P\left(>T_{L}\right) \propto T_{L}^{-\alpha}
$$


Fig. 3.8 Log-log cumulative distribution of cancellation orders lifetime. The grey line represents theoretical distribution estimated from Fig. 3.5 using Eq. (3.4) and the black line shows real data cancellation lifetime (Fig. 3.4)

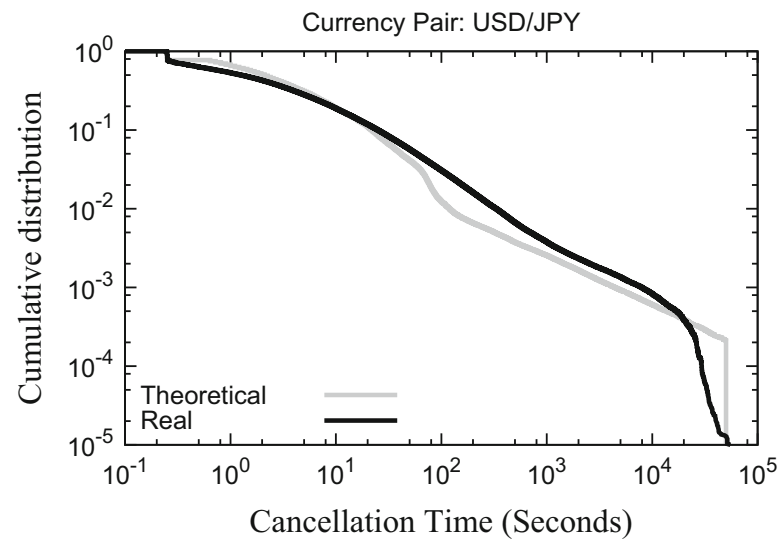

In addition, cancellation orders from mid-price follows:

$$
P(>\bar{r}) \propto \bar{r}^{-\delta}
$$

By incorporating Eqs. (3.2), (3.5) and (3.6) we have the following relationship for cancellation lifetime cumulative distribution:

$$
P\left(>T_{L}\right) \propto T_{L}^{-\frac{\delta}{2}}
$$

Therefore, we find that $\delta$ can be linked with $\alpha$ :

$$
\frac{\delta}{2} \approx \alpha
$$

\subsection{Cancellation Orders and Market Price Movement}

One might suspect that market participants cancel their orders depending on the market conditions. To analyze the relative behavior of market participants toward market price movements, we introduce new quantities $A_{i}$ and $B_{i}$ as shown below. The numerator of both quantities represents the distance of an order from the midprice at annihilation (time T). $m(t)$ is the mid-price at time $\mathrm{t}, b_{i}(t)$ refers to the bid order $i$-th submitted at time $t$ and $a_{i}(t)$ refers to the ask order $i$-th submitted at time $t$. The denominator represents the distance of the order from the mid-price at injection (time t).

In the case that $A_{i}=0\left(B_{i}=0\right)$ means that the mid-price did not move from the injection of the $i$-th ask (bid) to its annihilation moment. $A_{i}\left(B_{i}\right)$ higher than zero means that the market movement moves further from the order price; it becomes more difficult for this order to be executed because the distance from the mid-price 
Table 3.2 Frequency of cancellation orders filtered by the ratio value

\begin{tabular}{l|l|l|l|l|l|l}
\hline Currency & Total & $>0$ & $<0$ & $=0$ & Neg. spread & Exclude \\
\hline USD/JPY & $2,436,385$ & $1,063,715$ & 877,185 & 457,397 & 7157 & 30,931 \\
\hline EUR/JPY & $1,439,801$ & 672,453 & 446,137 & 315,960 & 429 & 4822 \\
\hline
\end{tabular}

Fig. 3.9 Log-log probability density function (PDF) of price change from initial distance for USD/JPY currency pair. Grey bars represent situations where the market price is getting further from individual orders price. Black bars represent situations where the market price is getting closer from individual orders price

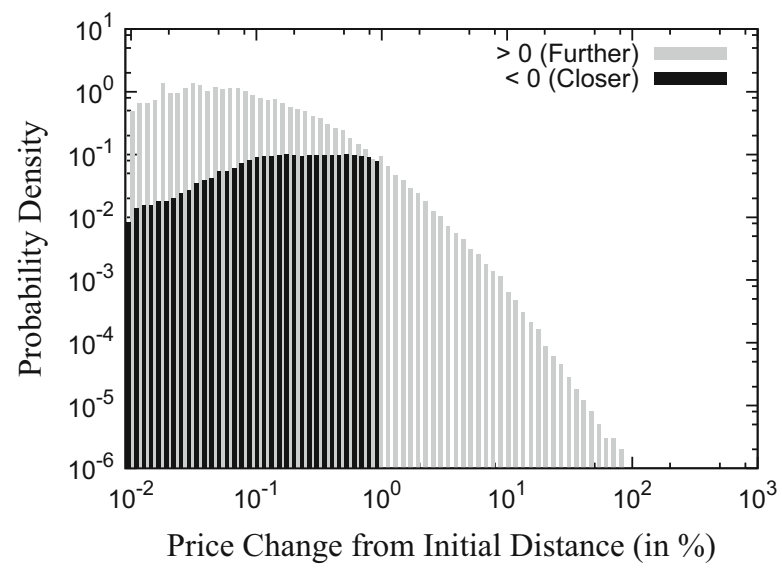

is getting higher. Inversely, $A_{i}\left(B_{i}\right)$ lower than zero means that the market moves closer toward the order price.

$$
\begin{aligned}
A_{i} & =\frac{\left[a_{i}(t)-m(T)\right]}{\left[a_{i}(t)-m(t)\right]}-1 \\
B_{i} & =\frac{\left[m(T)-b_{i}(t)\right]}{\left[m(t)-b_{i}(t)\right]}-1
\end{aligned}
$$

Table 3.2 describes the frequency of cancellations filtering for different situations. The label Neg. Spread means that the bid-ask spread is negative at the moment of the cancellation order (time T). Again, it can happen because EBS market requires a credit agreement between every market participant before realizing transactions. If there is no agreement, even a buying price higher than a selling price will not trigger a transaction. In our probability density function analysis, we do not include this type of data. The label Exclude represents situations where the injection of order was initially done at a negative spread (time t). This situation has been excluded to the probability density function analysis.

To construct Fig. 3.9, we use bin size of $10^{-0.3}$. Bars with label $>0$ (Further) represent cases where the ratio of Eqs. (3.9) and (3.10) is higher than zero. Inversely, bars with label $<0$ (Closer) represent cases where the ratio of Eqs. (3.9) and (3.10) is lower than zero. 


\subsection{Discussion}

Our study focuses on lifetime of annihilated orders either by execution or cancellation. Using the example of USD/JPY currency pair, we demonstrate the scaling relation between the distance from mid-price and the random walk theory and compare the result with real data. The non-trivial difference between cancellation and execution lifetime can be explained by a non-random cancellation process. The EBS database especially helps us to discover that cancellation probability depends on the distance of those orders from the mid-price.

In addition, we have seen that market participants have a non-symmetric cancellation behavior, which depends on the movement of the market price. Further research linking the impact of market movement (volatility) and cancellation behavior may contribute to quantify the impact of cancellation orders on market stability. It may represent promising future research subjects.

Open Access This book is distributed under the terms of the Creative Commons Attribution Noncommercial License which permits any noncommercial use, distribution, and reproduction in any medium, provided the original author(s) and source are credited.

\section{References}

1. Bachelier L (1900) Annales Scientifiques de lÉcole Normale Supérieure 17:21

2. Eisler Z, Bouchaud JP, Kockelkoren J (2010) Quant Finan 12:1395

3. Zovko I, Farmer JD (2002) Quant Finan 2:387

4. Challet D, Stinchcombe R (2002) http://arxiv.org/abs/cond-mat/0208025

5. Takayasu M, Watanabe T, Takayasu H (2010) Approaches to large-scale business data and financial crisis. Springer, Tokyo

6. Hasbrouck J (2007) Empirical market microstructure. Oxford University Press, New York

7. Mantegna RN, Stanley HE (2000) An introduction to econophysics. Cambridge University Press, Cambridge

8. Yura Y, Takayasu H, Sornette D, Takayasu M (2014) Phys Rev Lett 112:098703

9. SEC (2013) The speed of the equity markets, 2013-05

10. SEC (2013) Quote lifetime distributions. Data Highlight 2013-04

11. SEC (2014) Equity market speed relative to order placement. Data Highlight 2014-02

12. Lauricella T, Patterson S (2010) SEC probes canceled trades. Wall Street Journal, Sept 12010

13. Menkveld AJ, Yueshen ZB (2013) http://ssrn.com/abstract $=2243520$

14. Menkveld AJ (2013) J Finan Mark 16:712-740 